\title{
Editorial
}

\section{The Epidemiology of Contact Transmission: Beyond Semmelweis}

\author{
Edward S. Wong, MD
}

In his article on the transmission of nosocomial infections, published in the Proceedings of the Third Decennial International Conference on Nosocomial Infections, sponsored by the Centers for Disease Control and Prevention (CDC) in 1991, Robert Weinstein placed hospital personnel in the center of the maelstrom of nosocomial transmission (Figure). ${ }^{1}$ He estimated that hospital personnel were responsible for approximately $20 \%$ to $40 \%$ of the nosocomial spread of pathogens from patient to patient through contact transmission. The conventional wisdom is that, during direct contact, the hands of personnel pick up exogenous organisms that are then deposited onto medical devices or wounds to cause infection. This mechanism of spread of infection is precisely what Semmelweis hypothesized when he observed the spread of puerperal sepsis. Since the Fourth Decennial Conference on Nosocomial Infections will be held shortly in Atlanta, Georgia, it is fair to ask whether we have learned anything new about contact transmission since the last decennial conference. How far beyond Semmelweis have we gone?

Semmelweis correctly postulated that the hands carried a potentially deadly agent, but he did not know that it was a microbiological agent. We now know that our hands are an ecosystem comprised of permanent or resident flora and transient flora. ${ }^{2,3}$ The permanent florae consist of proprionibacteria, corynebacteria, micrococci and other staphylococcal species, including Staphylococcus epidermidis, Staphylococcus hominis, Staphylococcus capitis, all residing in stratum corneal layer, feeding on lipids and cellular debris. Permanent florae are viewed as "good" florae, as they rarely lead to disease; and, by their production of lipids and bacteriocins, permanent florae act to resist colonization by other microorganisms that potentially are more pathogenic. Transient florae do not normally reside on the skin, but are picked up during direct contact with patients or contaminated fomites. Unless they are eradicated through hand washing, they can be passed on or shed onto wounds, where, because of their pathogenic potential, they can lead to infection.

What do we know about the epidemiology of hand contamination, since this is the critical first step in the process of cross-infection? Are certain patient-care activities more prone to result in hand contamination? Is there a critical inoculum that is more likely to lead to infection versus colonization? During the 1970 s, the Fulkerson scale was developed and used to rank nursing activities according to their potential for hand contamination. ${ }^{4}$ Activities, ranked from 1 to 7 , ranged from contact with cleaned or washed materials down to contact with objects in contact with patient secretions. These were considered "clean" activities. "Dirty" activities included contact with uninfected patient secretions, infected secretions or excretions, materials contaminated with secretions or excretions, or the infected sites themselves. The purpose of the Fulkerson scale was to identify nursing activities that require hand washing. The presumption was that dirtier activities led to higher rates of cross-infection, but this has never been shown.

Recently, Pittet and coworkers published studies that add to and further refine our knowledge of hand contamination. ${ }^{5,6}$ Pittet and coworkers reconfirmed that the type of patient contact activity was important in determining the degree of hand contamination. In their study, respiratory care qualitatively resulted in a greater degree of hand contamination than handling of body fluid secretions (uninfected), which in turn posed a greater risk of contamination than skin contact. They also were able to demonstrate 


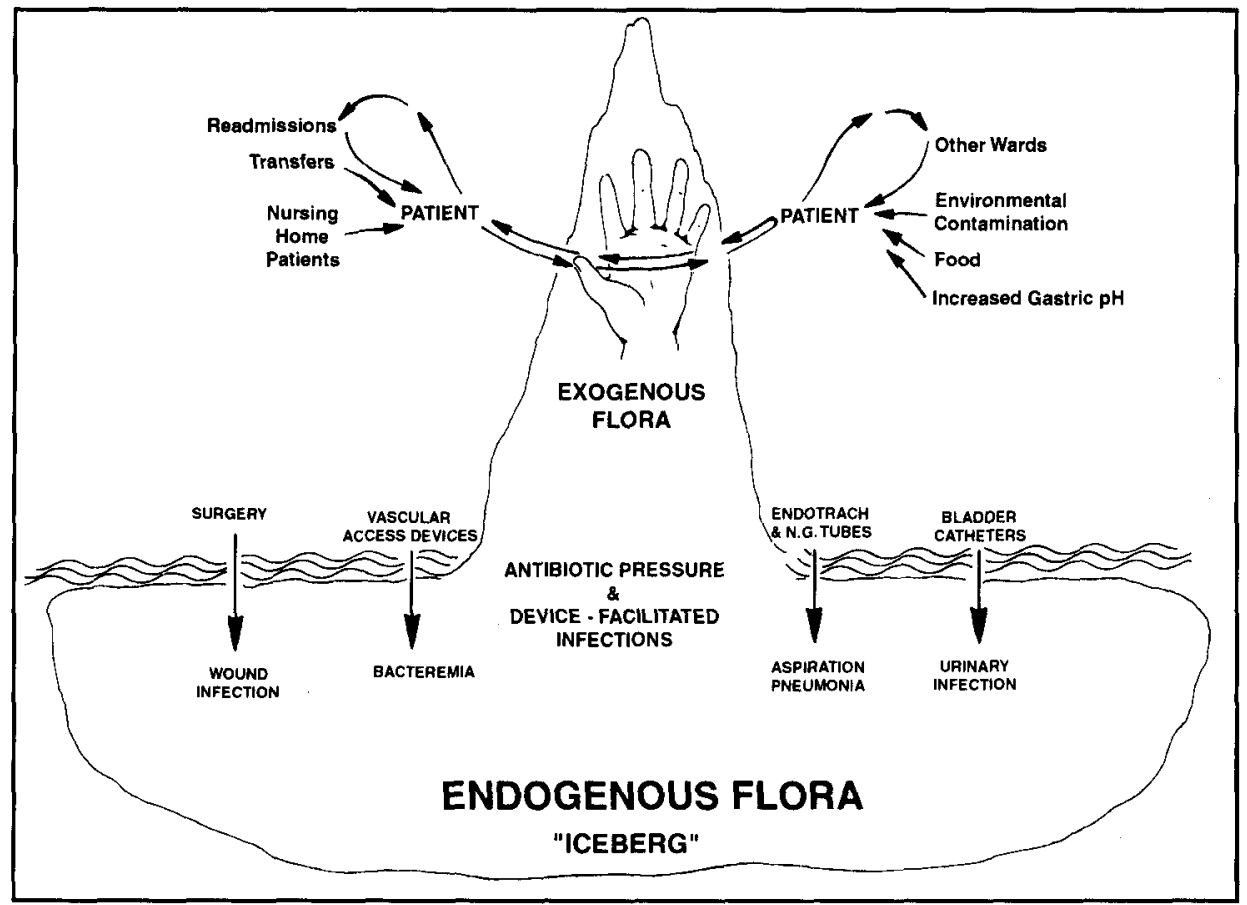

FIGURE. Dynamics of the transmission of nosocomial pathogens in the intensive care unit. Abbreviation: NG, nasogastric. Reprinted with permission from Excerpta Medica Inc. Weinstein RA. Epidemiology and control of nosocomia infections in adult intensive care units. Am J Med 1991;91(suppl 3B):179S$184 S^{1}$

that each minute of respiratory care, contact with secretions, and skin contact added another 21 colony-forming units (CFU), $16 \mathrm{CFU}$, and $4 \mathrm{CFU}$, respectively, to the degree of hand contamination. Thus, the authors added another dimension to the equation, the importance of the duration of activity. While contact with the noninfected intact skin of a patient may seem innocuous enough, the inference is that, given enough time, clinically significant cumulative hand contamination will occur. It is intriguing to learn that 5 minutes of skin contact will result in the same degree of contamination as 1 minute of respiratory care.

The study also documented that glove use protects the hands of the healthcare worker from bacterial contamination. ${ }^{5}$ The slope of acquisition of bacteria for gloved hands was flat, compared to the steady rise in the concentration of bacteria for ungloved hands. This finding is reassuring, given our reliance on gloving as a central element in any of the currently available systems of isolation (eg, the Body Substance Isolation system of Lynch and Jackson ${ }^{7}$ or the CDC's Standard Precautions). ${ }^{8}$

In this issue of Infection Control and Hospital Epidemiology, Moolenaar and coworkers identify yet another risk factor that has a potential impact on hand contamination and cross-infection. ${ }^{9}$ In their investigation of bloodstream infections and endotracheal tube colonization by Pseudomonas aeruginosa, the authors incriminated two nurses in a neonatal intensive care unit whom they believe were the sources of the epidemic organism. The evidence linking the two nurses to the outbreak included the following: (1) microbiological evidence: culture of their hands yielded the epidemic organism; (2) genetic evidence: the isolates recovered from nurses' fingertips were identical by pulsed-field electrophoresis; and (3) epidemiological evi- dence: case-control analysis demonstrated a significant association between exposure to these two nurses and acquiring $P$ aeruginosa.

Notable and novel was their observation that both nurses had long fingernails, one natural and the other artificial. When hand-culture results were analyzed, the authors noted that nurses with long fingernails were more likely to be positive for Pseudomonas than nurses with short- to medium-length fingernails ( 2 of 12 vs 1 of 80 ; relative risk, $0.08 ; P<.05)$. The association of long nails with colonization and spread of Pseudomonas was deemed sufficiently strong for the investigators to implement a new policy restricting nurses in the neonatal intensive care unit (NICU) from wearing long (either natural or artificial) fingernails as an infection control measure. How effective was this new policy? Well, the number of cases in fact did diminish, but the problem did not entirely go away. This suggests perhaps that other factors might be involved. Indeed, when the hand-culture data were reevaluated by restricting the analysis only to nurses with patient-care responsibilities, the association between colonization with Pseudomonas and long nails was no longer significant. The case against long fingernails could have been made stronger if serial hand cultures had been obtained and had demonstrated that colonization with Pseudomonas persisted longer in nurses with long versus short or medium fingernails.

For any epidemiological association noted, one always has to ask the question whether the association makes biological sense. Therefore, how do long fingernails permit prolonged bacterial colonization? McGinley and coworkers previously demonstrated that the subungual space under the fingernails harbors the greatest concentration of microorganisms in the hand..$^{10}$ It could be postu- 
lated that longer nails might provide an even more hospitable environment for microorganisms because of increased moisture, cellular debris, and lipid content. In addition, longer nails could lead to prolonged colonization if their presence somehow interfered with correct handwashing techniques. One could image that having long nails might disrupt the flow of handwashing movements, preventing the fingertips from being adequately scrubbed.

What are the implications of Moolenaar's and coworkers' findings? Is the evidence sufficiently strong for us to ban personnel from wearing long natural or artificial nails as a matter of policy? Based on the data presented, I do not believe so. We clearly need further corroborating evidence. Noteworthy are the results of an investigation, presented recently at the Infectious Disease Society of America's annual meeting, of an outbreak of $P$ aeruginosa in an NICU. ${ }^{11}$ This investigation incriminated artificial nails as a risk factor for hand carriage among NICU personnel. In the parlance of the $\mathrm{CDC}$ guidelines, if a recommendation against the use of long or artificial nails was ever to be made, such a policy would have to be ranked as a category II recommendation, that is, one that has the backing by some studies but not by definitive studies. A category II recommendation might be useful for some hospitals but, as a rule, is not recommended for adoption by all hospitals.

\section{REFERENCES}

1. Weinstein RA Epidemiology and control of nosocomial infections in adult intensive care units. Am J Med 1991;91(suppl 3B):179S-184S.

2. Price PB. The bacteriology of normal skin: a new quantitative test applied to a study of the bacterial flora and the disinfectant action of mechanical cleansing. J Infect Dis 1938:63:301-318.

3. Woodroffe RC, Shaw DA. Natural control and ecology of microbial populations on skin and hair. In: Skinner FA, Carr JG, eds. The Normal Microbial Flora of Man. London, UK: Academic Press; 1974:3-34.

4. Fox MK, Langner SB, Well RW. How good are hand washing practices? Am I Nurs 1974;74:1676-1678.

5. Pittet D, Dharan S, Touveneau S, Sauvan V, Perneger TV. Bacterial contamination of the hands of hospital staff during routine patient care. Arch Intern Med 1999;159:821-826.

6. Harbarth S, Sudre P, Dharan S, Cadenas M, Pittet D. Outbreak of Enterobacter cloacae related to understaffing, overcrowding, and poor hygiene practices. Infect Control Hosp Epidemiol 1999;20:598-603.

7. Lynch P, Jackson MM, Cummings MJ, Stamm WE. Rethinking the role of isolation practices in the prevention of nosocomial infections. Ann Intern Med 1987;107:243-246.

8. Garner JS, the Hospital Infection Control Practices Advisory Committee. Guideline for isolation precautions in hospitals. Infect Control Hosp Epidemiol 1996;17:53-80.

9. Moolenaar RL, Crutcher JM, San Joaquin VH, Sewell LV, Hutwanger LC, Carson LA, et al. A prolonged outbreak of Pseudomonas aeruginosa in a neonatal intensive care unit: did staff fingernails play a role in disease transmission? Infect Control Hosp Epidemiol 2000;21:80-85.

10. McGinley KL, Larson EL, Leyden JJ. Composition and density of microflora in the subungual space of the hand. $J$ Clin Microbiol 1988;26:950-953.

11. Jakob KM, Foca M, Whittier S, Della-Latta P, Saiman L. Risk factors for Pseudomonas aeruginosa hand carriage in healthcare workers (HCW) during an investigation of increased infection in a neonatal intensive care unit (NICU). Presented at the 37th Annual Meeting of the Infectious Diseases Society of America; November 18-21, 1999; Philadelphia, PA. Abstract 21. 